\title{
On Lowen's fuzzy compact spaces
}

\author{
Francisco Gallego Lupiáñez
}

\begin{abstract}
In this paper, we obtain an axiomatic characterization of Lowen's fuzzy compactness.
\end{abstract}

Keywords- Mathematics, fuzzy sets, Topology, Lowen's compactness, operators.

\section{INTRODUCTION}

There exist various notions on compactness in fuzzy topological spaces (see the books by Liu and Luo [1]or Palaniappan [5]).

This fact is due to problems as the conservation of compactness fuzzy notions by products, or the other problem on some kinds of fuzzy compactness which are good extensions of compactness and other are not. The concept of fuzzy compactness defined by R. Lowen is a good extension of compactness and this notions is productive.

On the other hand there exists an interesting characterization of compact Hausdorff topological spaces (due to De Groot) using five axioms.

In this paper, we obtain an axiomatic characterization of Lowen's fuzzy compactness.

\section{BASIC DEFINITIONS}

We use fuzzy topological spaces in Lowen's sense [3].

First, we present some basic definitions:

Definition 1. I denotes the unit interval, and $I_{r}$ is $I$ equipped with the topology

$\left.\left.T_{r}=\{] \alpha, 1\right] \mid \alpha \in I\right\} \cup\{I\}$.

Definition 2. [2] If $\delta$ is a fuzzy topology on $X$ then $\imath(\delta)$ is the initial topology on $X$ for the family of functions $\delta$ and the topological space $I_{r}$.

Definition 3. [2] Let Top denote the category of topological spaces and Fuz the category of fuzzy topological spaces. We define the functor $\omega: \mathbf{T o p} \rightarrow \mathbf{F u z}$ which to each object $(X, T)$ associates $(X, \omega(T))$ where $\omega(T)$ is the fuzzy topology consisting of all continuos functions from $(X, T)$ to $I_{r}$. A fuzzy topological space $(X, \omega(T))$ is called topologically generated.

Definition 4. When introducing a generalization, in $\mathbf{F u z}$, of a notion in Top it is natural to request of the generalization that when restricted to Top it should give the original notion. Such an extension is called a good extention ([4]).

Definition 5. [3] A fuzzy set $v$ is fuzzy compact if for all family $\beta$ of open fuzzy sets such that $\sup _{\mu \in \beta} \mu \geq v$ and for all $\varepsilon>0$, there exists a finite subfamily $\beta_{0} \subset \beta$ such that $\sup _{\mu \in \beta_{0}} \geq v-\varepsilon$.

Definition 6. [3] The fuzzy topological space $(X, \delta)$ is fuzzy compact if each constant fuzzy set in $(X, \delta)$ is fuzzy compact. 
III. RESULTS

Theorem. Let $\Gamma$ be a class of fuzzy topological spaces which satisfies the following conditions:

(a) The topological product of any family of members of $\Gamma$ is a member of $\Gamma$.

(b) Every closed fuzzy subspace of a member of $\Gamma$ is a member of $\Gamma$.

(c) If $X \in \Gamma$ and $Y \in \Gamma$ and if $X$ is a fuzzy subspace of $Y$, then $X$ is a closed fuzzy subspace of $Y$.

(d) Every closed fuzzy continuous image of a member of $\Gamma$ is a member of $\Gamma$.

(e) The class $\Gamma$ contains a fuzzy topological space consisting of more than one point.

Then, $\Gamma$ is precisely the class of all fuzzy compact $T_{2}$ spaces.

Proof. Let

$\Gamma^{*}=\{(X, T)$ fuzzy topological space /

$(X, \omega(T)) \in \Gamma$, i.e.

$\Gamma^{*}=\{(X, T) \mid T=\imath(\tau),(X, \tau) \in \Gamma\}$.

i) Let $\left\{\left(X_{j}, T_{j}\right)\right\}_{j \in J} \subset \Gamma^{*}$ then

$T_{j}=\imath\left(\tau_{j}\right)$ for every $j \in J$ such that

$\left(X_{j}, \tau_{j}\right) \in \Gamma$. Then,

by (a), $\left(\prod X_{j}, \prod \tau_{j}\right) \in \Gamma$ and

$\left(\prod X_{j}, l\left(\prod \tau_{j}\right)\right) \in \Gamma^{*}$. Thus

$\left(\prod X_{j}, \prod l\left(\tau_{j}\right)\right)=\left(\prod X_{j}, \prod T_{j}\right) \in \Gamma^{*}$

ii) Let $Y \subset X$, such that $Y$ is closed in $(X, T)$. Then $Y$ is closed fuzzy in $(X, \tau) \in \Gamma$. From (b), the fuzzy subspace $\left(Y, \tau_{\mid Y}\right) \in \Gamma$, and $\left(Y, l\left(\tau_{\mid Y}\right)\right) \in \Gamma^{*}$, i.e. $\left(Y, l(\tau)_{\mid Y}\right)=\left(Y, T_{\mid Y}\right) \in \Gamma^{*}$.

iii) Let $(X, T),(Y, S) \in \Gamma^{*}$ such that $X$ is a subspace of $Y$. Then, $X$ is a fuzzy subspace of $(Y, \omega(S)) \in \Gamma$, and, by (c), $X$ is a closed fuzzy of $(Y, \omega(S))$.Thus $X$ is closed in $(Y, S)$.

iv) Let an onto closed continuous map $f:(X, T) \rightarrow(Y, S)$, such that $(X, T) \in \Gamma^{*}$. Then, if $\tau=\omega(T)$, and $\varsigma=\omega(S)$, we have an onto closed continuous fuzzy map $f:(X, \tau) \rightarrow(Y, \varsigma)$, with $(X, \tau) \in \Gamma$. By $(\mathrm{d}),(Y, \varsigma) \in \Gamma$.Then $(Y, S) \in \Gamma^{*}$.

v) From (e) there exists a space $(X, \tau) \in \Gamma$ such that $X$ has of more than one point. Then $(X, \imath(\tau)) \in \Gamma^{*}$ and $X$ has more than one point.

From a Theorem of de Groot (see [6]) it follows that $\Gamma^{*}$ is precisely the class of all compact Hausdorff topological spaces. Thus $\Gamma$ is the class of all compact Hausdorff fuzzy topological spaces.

\section{REFERENCES}

[1] Liu Y.-M and Luo M.-K. , Fuzzy Topology, World Scientific (Singapore), 1997.

[2] R.Lowen, "Topologies flous", C.R. Acad. Sc. Paris, ser. A, Vol 278, pp. 925-928, 1974.

[3] R. Lowen, " Fuzzy topological spaces and fuzzy compactness", J. Math. Anal. Appl., Vol 56, pp.621633.1976.

[4] R. Lowen, "A comparison of different compactness notions in fuzzy topological spaces" J. Math. Anal. Appl. Vol 64, pp. 446-454, 1978.

[5] N. Palaniappan, Fuzzy Topology, CRC Press, Boca Raton, FL, 2002

[6] E. Wattel, The compactness operator in Set-Theory and Topology, Math. Centr. (Amsterdam), 1979.

Francisco Gallego Lupiáñez has born in 1958, in Madrid, Spain. He received the B.S. and Ph.D. degrees of the University Complutense, Madrid, in 1980 and 1985, respectively.

Since 1981, he has been with the Department of Geometry and Topology, University Complutense, where he is currently an Associate Professor. His research interests include General Topology and Fuzzy Topology. 